\title{
Three-year clinical outcomes of a sirolimus-eluting bioresorbable scaffold (XINSORB) and a metallic stent to treat coronary artery stenosis
}

\author{
Yizhe Wu\#, Zhifeng Yao", Jiasheng Yin", Jiahui Chen, Juying Qian, Li Shen, Lei Ge, Junbo Ge; on behalf \\ of the XINSORB randomized clinical trial
}

Shanghai Institute of Cardiovascular Diseases, Department of Cardiology, Zhongshan Hospital, Fudan University, Shanghai, China

Contributions: (I) Conception and design: L Shen, L Ge, J Ge; (II) Administrative support: J Qian, J Ge; (III) Provision of study materials or patients:

Y Wu, Z Yao; (IV) Collection and assembly of data: J Chen, Z Yao; (V) Data analysis and interpretation: Y Wu, J Yin, L Ge; (VI) Manuscript writing:

All authors; (VII) Final approval of manuscript: All authors.

\#These authors contributed equally to this work.

Correspondence to: Junbo Ge; Lei Ge; Li Shen. 180 Fenglin Road, Shanghai 200032, China. Email: ge.junbo2@zs-hospital.sh.cn;

ge.lei@zs-hospital.sh.cn; nt_sunny@163.com.

\begin{abstract}
Background: Recent studies have shown increased risks of late target lesion failure (TLF) and thrombosis using a bioresorbable scaffold (BRS). However, the results of the ABSORB China study offered a different means of understanding the long-term performance of BRSs. We tested the 3-year clinical outcome of the XINSORB BRS in a multicenter, randomized controlled clinical trial (ChiCTR1800014966).

Methods: Eligible patients with one or two de novo coronary lesions were randomly assigned 1:1 to be treated with XINSORB scaffolds and metallic sirolimus-eluting stents (SESs). The clinical endpoints include TLF [cardiac death, target vessel-related myocardial infarction (TV-MI), or ischemia-driven target lesion revascularization (ID-TLR)], its components, and devised thrombosis.

Results: Three hundred ninety-five patients were enrolled and randomized to the XINSORB (N=200) and SES $(\mathrm{N}=195)$ arms. The clinical 3-year follow-up included $95.5 \%$ of the XINSORB-treated patients and $92.8 \%$ of the SES-treated patients. Dual antiplatelet therapy was at $59.0 \%$ of the XINSORB-treated and $52.8 \%$ of the SES-treated patients $(\mathrm{P}=0.34)$. There were no significant differences in the clinical outcomes between the XINSORB and SES arms, including in TLF (4.0\% vs. 6.2\%, $\mathrm{P}=0.29)$, cardiac death $(1.0 \%$ vs. $0 \%, \mathrm{P}=\mathrm{NA})$, TV-MI (1.0\% vs. $0 \%, \mathrm{P}=\mathrm{NA})$, and ID-TLR (3.5\% vs. $6.2 \%, \mathrm{P}=0.19)$. The rate of confirmed/ probable device thrombosis in the XINSORB-treated patients was only $1.0 \%(2 / 200)$.

Conclusions: In this XINSORB randomized clinical trial, the XINSORB scaffolds and SESs showed similar efficacy and safety up to the 3-year follow-up. The rates of TLF and device thrombosis were low and comparable between the two arms.
\end{abstract}

Keywords: Coronary artery disease; percutaneous coronary intervention; bioresorbable scaffold (BRS)

Submitted Aug 12, 2020. Accepted for publication Nov 06, 2020.

doi: 10.21037/atm-20-6739

View this article at: http://dx.doi.org/10.21037/atm-20-6739

\section{Introduction}

Bioresorbable scaffolds (BRSs) (mainly referred to as BVS, Abbott Vascular, Santa Clara, CA, USA) showed favorable outcomes at a 1-year follow-up cobalt-chromium everolimus-eluting stent (XIENCE, Abbott Vascular, Santa
Clara, CA, USA) (1). However, there were concerns that scaffold thrombosis and target lesion failure (TLF) after treatment with BVS increased beyond the 2-year followup (2-4). Yet to the results of ABSORB studies performed outside of China, the results from the ABSORB China 
study showed excellent clinical outcomes of treatment with BVS observed at three years. The rates of TLF (5.5\% for BVS and $4.7 \%$ for XIENCE) and device thrombosis ( $0.9 \%$ for BVS and $0.0 \%$ for XIENCE) were low and comparable between the treatment arms $(5,6)$. The XINSORB BRS (Shandong, Huaan, China) was a new device compared with BVS, and we have previously reported the first performance of the device $(7,8)$. The long-term results of the XINSORB BRS in a first-in-human study were promising and showed acceptable efficacy with relatively low rate of scaffold thrombosis (9). The randomized control clinical trial of the XINSORB scaffold started in October 2014. The primary endpoint of one-year in-segment late luminal loss (LLL) of XINSORB was noninferior to the traditional metallic TIVOLI $^{\circledR}$ sirolimus-eluting stent (SES) $(0.19 \pm 0.32$ vs. $\left.0.31 \pm 0.41 \mathrm{~mm}, \mathrm{P}_{\text {noninferior }}=0.003\right)$. The rate of TLF was favorable for the XINSORB scaffold (2.5\% for XINSORB and $5.1 \%$ for SES, $\mathrm{P}=0.17$ ) (10). As a result, the XINSORB BRS was approved by China Food and Drug Administration in March 2020. Here, we report our analysis of the clinical results of the randomization part of the XINSORB clinical trial for up to three years. We present the following article in accordance with the CONSORT reporting checklist (available at http://dx.doi.org/10.21037/atm-20-6739).

\section{Methods}

\section{Study devices}

The XINSORB scaffold is composed of poly-L-lactic acid (PLLA). The design of the scaffold is like the ABSORB scaffold. Poly-D-L-lactic acid (PDLLA) mixed with PLLA carrying sirolimus is coated on the struts. The dosage of sirolimus ranges from $8-16 \mu \mathrm{g} / \mathrm{mm}$, depending on the length of the scaffold. The thickness of the strut is $160 \mu \mathrm{m}$.

The commercially available TIVOLI $^{\circledR}$ stent (Essen Technology, Beijing, China) is a cobalt-chromium SES with a biodegradable polymer. The efficacy and safety of this metallic stent have been confirmed in clinical trials $(11,12)$.

\section{Protocol, patient population, intervention, and follow-up}

We previously reported the study design of the XINSORB randomized clinical trial (10). The study was registered at the official website of the China Clinical Trial Registry (ChiCTR1800014966). The study included a randomization part and a registry part. Briefly, the randomization part of the study was a prospective, randomized, multicenter trial. The study complied with the Good Clinical Practice guidelines of the China Food and Drug Administration. All procedures performed in this study involving human participants were in accordance with the Declaration of Helsinki (as revised in 2013). The study was approved by Zhongshan Hospital (No. 2014-44). Written informed consent was obtained before patient enrollment. Eligible patients were randomized at a 1:1 ratio to receive a XINSORB scaffold or SES. A maximum of 2 de novo lesions in different native coronary arteries is included. The lesions were visually assessed to be less than $24 \mathrm{~mm}$, and the reference vessel diameter was from $2.75-3.5 \mathrm{~mm}$. The percentage of stenosis was from $50 \%$ to $100 \%$, with thrombolysis in myocardial infarction (TIMI) flow grade greater than 1 . The procedures were performed through the standard process. Both predilation of the target lesion and the post-dilation of the implanted scaffold are mandatory. Post dilation for metallic stents was recommended. If the post-dilation of a XINSORB scaffold was needed, the diameter of the non-compliant balloon was required to be shorter and a maximum of $0.25 \mathrm{~mm}$ larger than the device. All patients received regular clinical follow-up examinations at 30, 90, 180, and 270 days and 1, 2, 3, 4, and 5 years. Patients were angiographically examined at one year after the index procedure. Follow-up examinations up to three years were completed at the time of the present report. Clopidogrel was prescribed for at least 12 months, and aspirin was continued for the duration of the study. On-site monitoring of $100 \%$ of the trial data was performed.

The study is registered at official website of China Clinical Trial Registry (ChiCTR1800014966).

\section{Endpoints and definitions}

The primary endpoint for randomization was angiographic in-segment LLL, defined as the difference in the minimal luminal diameter from post-procedure to 12 months, to show the noninferiority of the XINSORB scaffold to the SES. The secondary endpoints included the deviceoriented composite endpoint (DoCE) of TLF [cardiac death, target vessel-related myocardial infarction (TV$\mathrm{MI}$ ), or ischemia-driven target lesion revascularization (IDTLR)], the patient-oriented composite endpoint (PoCE; all-cause death, all MI, or all revascularization), target vessel failure (TVF) [cardiac death, MI, or ischemia-driven target vessel revascularization (ID-TVR)], major adverse cardiac events (MACE) (cardiac death, MI, or ID-TLR), 
the individual component endpoints of these endpoints, and device thrombosis. Device thrombosis was defined as acute (<24 hours), subacute (1 to 30 days), late (30 days to 1 year), and very late (beyond one year), and the level of evidence (definite or probable) was on the Academic Research Consortium definition (13). An independent clinical event committee watched all clinical events.

\section{Statistical analysis}

All analyses were performed according to the intention-totreat (ITT) principle. Continuous variables are presented as the mean $\pm \mathrm{SD}$, and categorical variables are presented as counts and percentages. Normally distributed student's $t$-test compared continuous variables. Categorical variables are compared using the chi-squared test or Fisher's exact test. The cumulative event rates of the 3-year clinical outcomes were calculated from Kaplan-Meier estimates and were compared with the log-ranking test. Cox proportionalhazard regression was used to find the hazard ratios (HRs) and corresponding $95 \%$ confidential intervals (CIs).

All statistical analyses were two-sided and performed with a significance level of 0.05 using SAS software, version 9.4 (SAS Institute, Cary, North Carolina).

\section{Results}

\section{Patients and baseline characteristics}

Between October 2014 and September 2015, 395 patients were randomized at 17 sites in China. None withdrew their informed consent to take part in this study. As shown in Figure 1, five patients in the XINSORB arm crossed over to the other arm because the scaffolds did not be delivered to target lesions. One patient in the SES arm received a non-study device. The per-treatment-evaluation (PTE) population thus comprised 389 patients in the randomization part (195 XINSORB and 194 SES). We reached 185,193 , and 191 patients in the XINSORB arm and 186, 186, and 181 patients in the SES arm at the 1-, 2-, and 3-year follow-ups. The clinical follow-up rates in the XINSORB arm at 1, 2, and 3 years were $92.5 \%, 96.5 \%$, and $95.5 \%$, respectively, and these rates were similar in the two randomized arms.

Baseline information of the patients, lesions, and procedures in the ITT population is shown in Table 1. The patient demographics, risk factors, and lesion characteristics were well-balanced between the two arms. We classified most lesions treated in this study as ACC/AHA A or B1 lesions (95.7\% for XINSORB and $92.8 \%$ for SES, $\mathrm{P}=0.57$ ). More lesions were predicated in the XINSORB arm than in the SES arm (99.0\% vs. 91.2\%, $\mathrm{P}=0.0001)$. Balloons used for predilation in the XINSORB arm were larger in diameter than those in the SES arm $(2.88 \pm 0.46$ vs. $2.77 \pm 0.45 \mathrm{~mm}$ $\mathrm{P}=0.01)$. The rate of post-dilation in the XINSORB arm was much higher than that in the SES arm $(94.8 \%$, vs. $73.1 \%, \mathrm{P}<0.001)$, although there was no difference regarding the size of the balloon or the pressure used for post-dilation.

There were 118 patients in the XINSORB arm (59.0\%) and 103 patients in the SES arm (52.8\%) who were still on dual antiplatelet therapy (DAPT) with aspirin and clopidogrel at 3-year follow-up $(\mathrm{P}=0.34)$.

\section{Clinical outcomes}

The 3-year endpoints of TLF (4.0\% and 6.2\%, P=0.29), PoCE $(8.5 \%$ and $8.7 \%, \mathrm{P}=0.86)$, MACE ( $4.0 \%$ and $6.2 \%, \mathrm{P}=0.29)$, and TVF (4.5\% and $6.7 \%, \mathrm{P}=0.31$ ) were comparable in the XINSORB and SES arms, respectively. Five patients died in the XINSORB arm. Two deaths were cardiac deaths; the first patient died 308 days after the index procedure because of unknown reasons, while the other died 462 days after the index procedure. This patient had received a XINSORB scaffold $(3.5 \mathrm{~mm} \times 18 \mathrm{~mm})$ in the proximal left circumflex artery on April 27, 2015. We observed a favorable result at the one-year routine angiographic follow-up per protocol. Recurrent chest pain was recorded on July 25, 2016. Repeated angiography showed thrombosis at the treated site, and a metallic stent was deployed. However, the patient died on August 1, 2016. In the SES arm, there was only one noncardiac death. There were no significant differences between the two arms in any part endpoints for up to 3 years (Table 2 and Figure 2).

\section{Device thrombosis}

The 3-year rates of device thrombosis were low and comparable between the XINSORB and SES arms $(1.0 \%$ and $0 \%, \mathrm{P}=0.5)$. Angiography confirmed both thrombosis events in the XINSORB arm. One patient suffered recurrent chest pain 92 days after scaffold deployment. Electrocardiography revealed Q-wave MI in the anterior wall. Emergent angiography was performed and showed thrombosis in the XINSORB-treated segment. We 


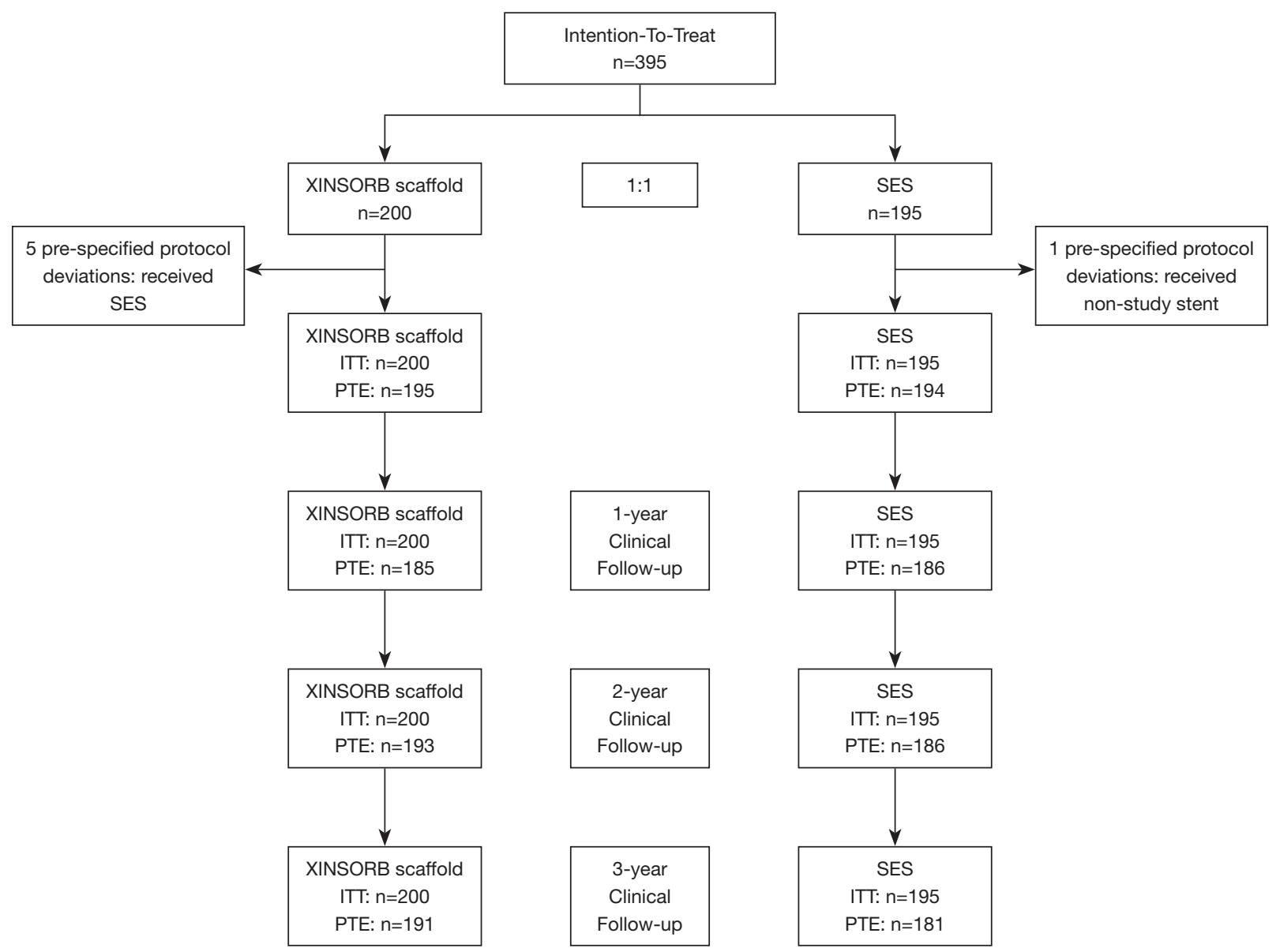

Figure 1 The flow chart of the XINSORB RCT. RCT, randomized control trial; ITT, intention-to-treat; PTE, per-treatment evaluation; SES, sirolimus-eluting stent.

deployed another metallic stent. The other thrombosis event was recorded 455 days after the index procedure, and the patient died as previously described. Both patients were still on DAPT at the time of the thrombosis event. We recorded no thrombosis events of 2 and 3 years after the index procedure.

\section{Discussion}

The main findings of this study were: (I) the XINSORB BRS was as effective as a traditional metallic SES for up to 3 years after the treatment of human coronary de novo lesions with moderate and simple complexity, and (II) the 3 -year rate of device thrombosis was low and comparable between the two devices.

Although significant achievements have been made in BRS design in the past decade, recently revealed results had increased concerns on the long-term risks of TLF and scaffold thrombosis using such devices (14-16). The 3 -year outcome of the ABSORB II study showed rates of TLF and device thrombosis of $10 \%$ and $3 \%$ for the BVS, respectively, which were significantly higher than the corresponding rates for the XIENCE stent $(5 \%$ and $0 \%)$. Similarly, the 3 -year results of the ABSORB III study showed a significantly higher rate of device thrombosis for the BVS than the XIENCE stent (2.3\% vs. $0.7 \%, \mathrm{P}=0.01)$, although the rate of TLF was comparable between the two devices $(13.4 \%$ vs. $10.4 \%, \mathrm{P}=0.055)$. However, the 3 -year results of the ABSORB China study were varied. The rates of TLF and definite/probable thrombosis in patients treated with the BVS were $5.5 \%$ and $0.9 \%$, respectively, which were like the rates in patients treated with the XIENCE stent (4.7\% and $0 \%$ ) (6). The rate of both TLF and device thrombosis at the 3 -year follow-up 
Table 1 Baseline patient and lesion characteristics, ITT population

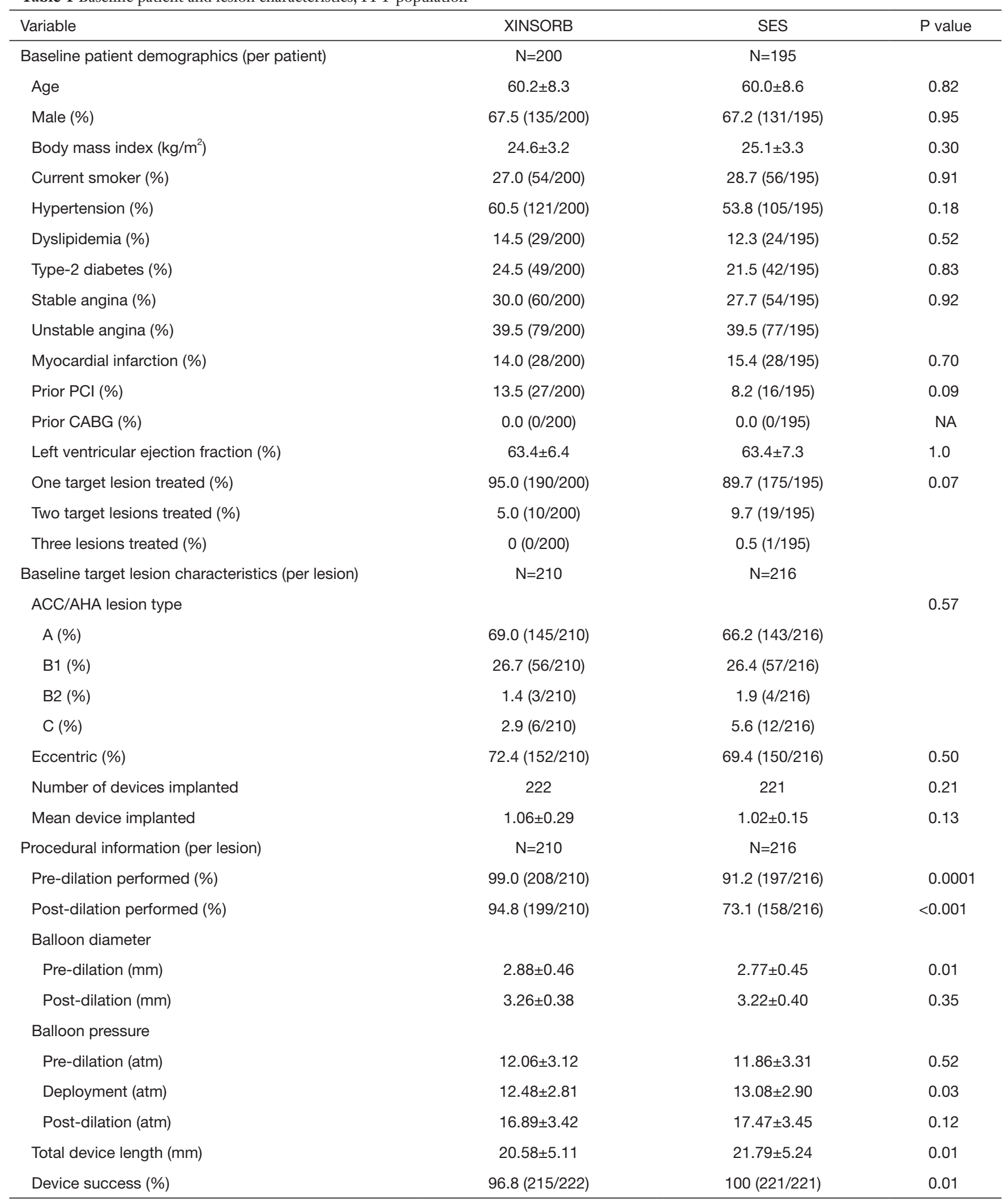

ITT, intention-to-treat; SES, sirolimus-eluting stent. 
Table 2 Three-year clinical outcomes, ITT population

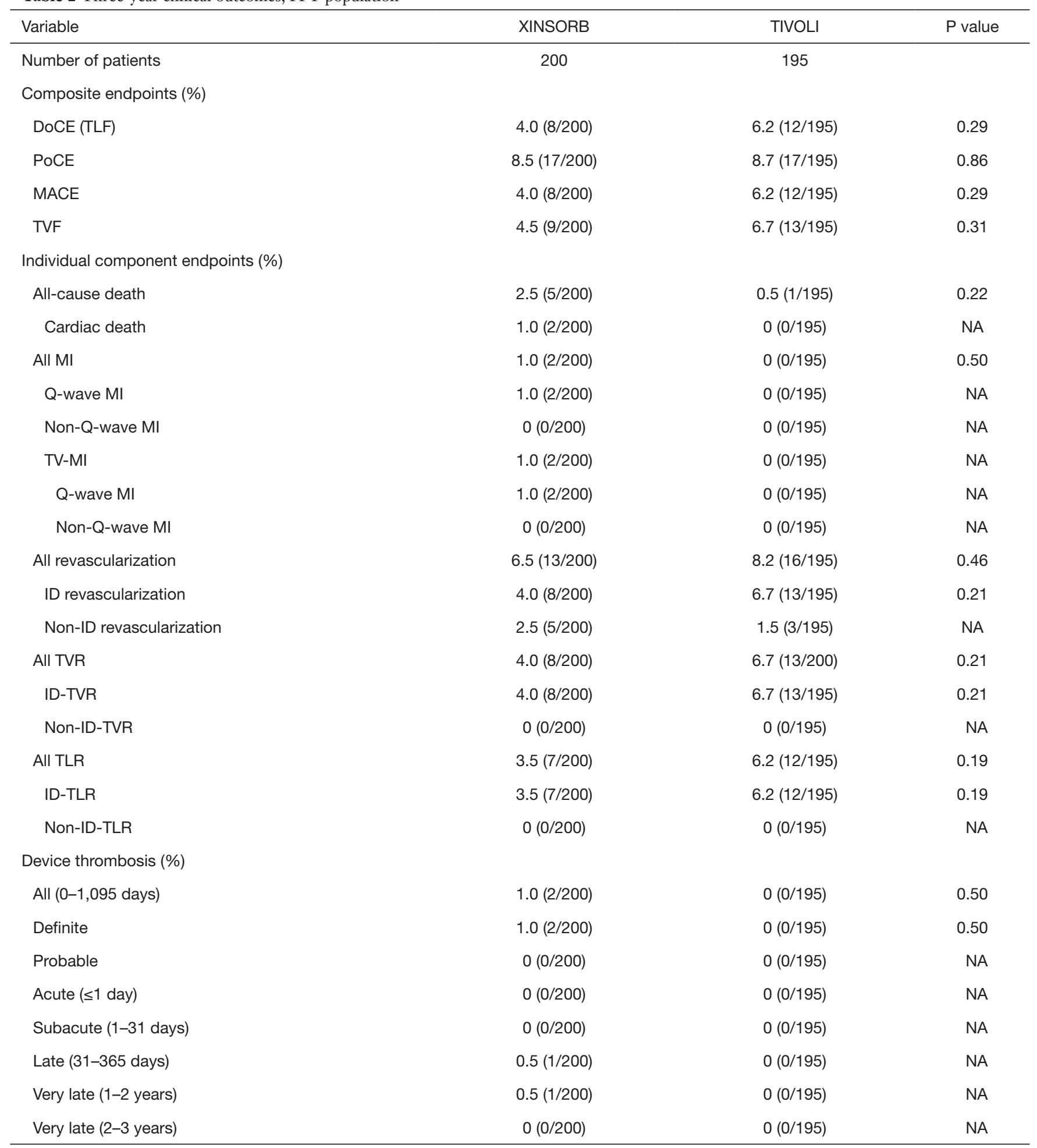

ITT, intention-to-treat; DoCE, device-oriented composite endpoint; PoCE, patient oriented composite endpoints; MACE, major adverse cardiac events; TVF, target vessel failure; MI, myocardial infarction; TV-MI, target vessel myocardial infarction; TVR, target vessel revascularization. 
A
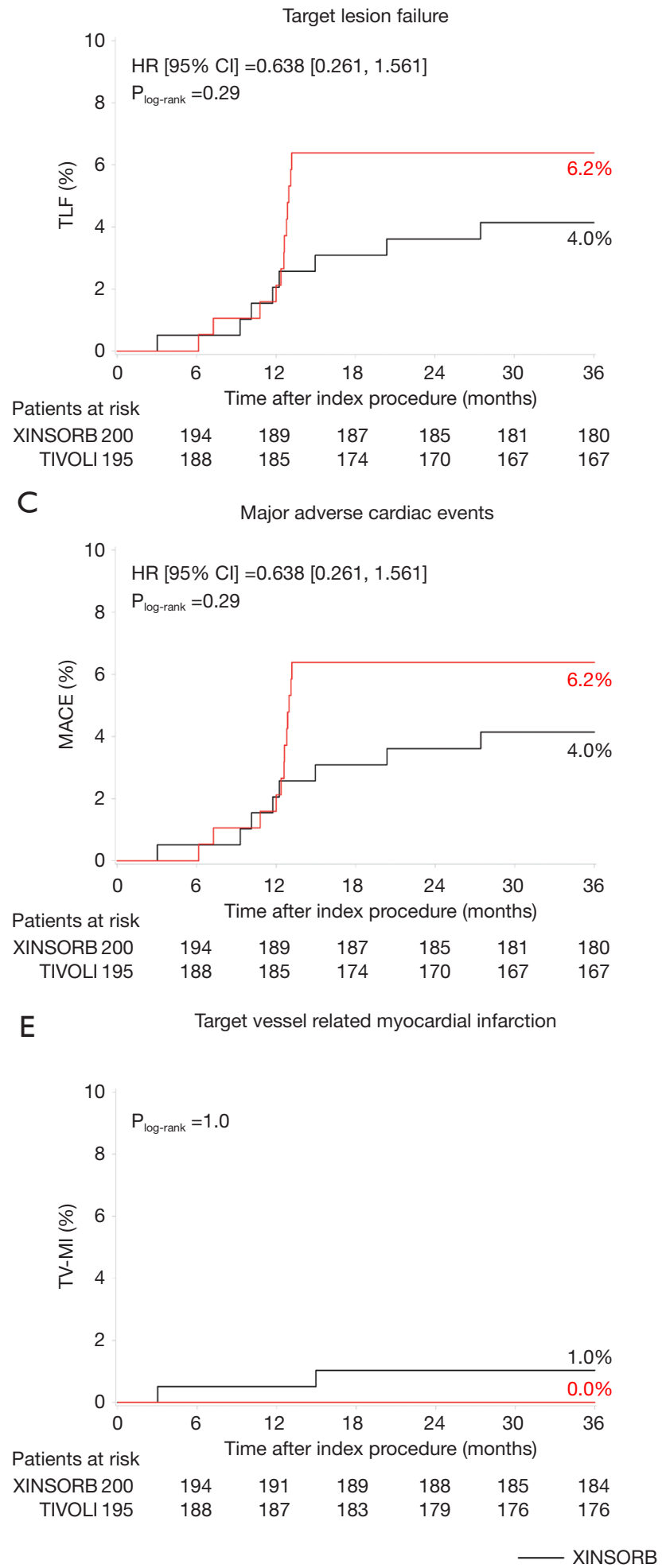

B

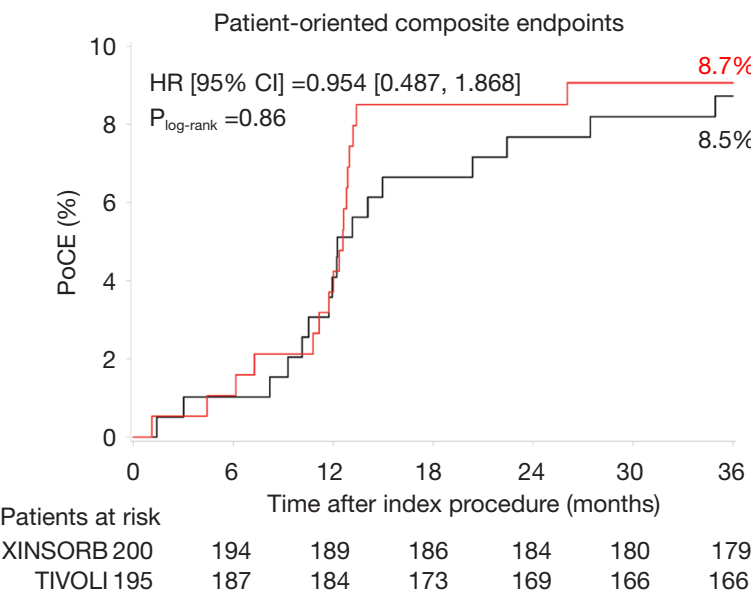

D

Ischemia-driven target lesion revascularization

10

$\mathrm{HR}[95 \% \mathrm{Cl}]=0.389[0.122,1.241]$

$8 \quad P_{\text {log-rank }}=0.19$

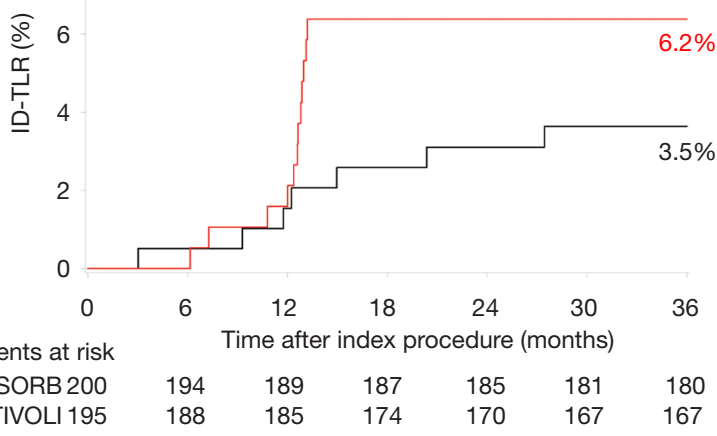

$\mathrm{F}$

All-cause death

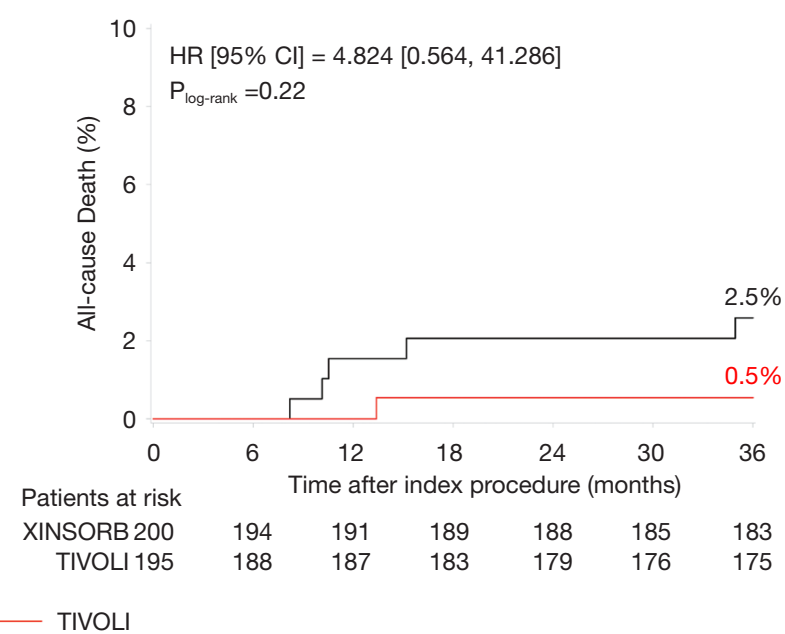

Figure 2 Kaplan-Meier curve of (A) TLF, (B) PoCE, (C) MACE, (D) ID-TLR, (E) TV-MI, and (F) all-cause death up to 3 year follow-up. TLF, target lesion failure; PoCE, patient oriented composite endpoints; MACE, major adverse cardiac events; ID-TLR, ischemia-driven target lesion revascularization; TV-MI, target vessel myocardial infarction. 
was significantly lower than the results retrieved outside of China. In our study, we found that 3-year rates of TLF and device thrombosis were low for the XINSORB scaffold and like those in the ABSORB China study. The PSP (preparing the lesion with optimal predilation, properly sizing the vessel, and optimal post-dilation with high pressure) technique was considered improving outcomes of BRS treatment. Data from the ABSORB IV study showed that treatment with the polymeric BVS using the PSP technique resulted in noninferior 30-day and 1-year TLF rates compared with treatment with the XIENCE stent (17). A reference vessel diameter less than $2.25 \mathrm{~mm}$ was a predictor of TLF (RR 1.72, 95\% CI: 1.28-2.32, $\mathrm{P}=0.0005$ ) and device thrombosis (RR 2.80, 95\% CI: 1.37-5.71, $\mathrm{P}=0.006)$. In the ABSORB China study, fewer small vessels (RVD $<2.25 \mathrm{~mm}$ ) $(9.6 \%)$ were treated with the BVS than in the ABSORB II (19.1\%) and ABSORB III (18.3\%) studies. In our study, most lesions treated with the XINSORB scaffold were predilated and post dilated, contributing to the favorable results. Long-term DAPT was associated with lower MACE and thrombosis rates $(18,19)$. Lactic acid resulting from biodegradation of the PLLA backbone is a sustainable inducer of vessel wall inflammation, which might cause scaffold thrombosis. Ideally, DAPT should be supported until full bioabsorption of the PLLA struts (20). However, only $29.8 \%$ of patients treated with the BVS in the ABSORB II study were on DAPT at the 3-year followup (21). In our study, 59\% of patients treated with the XINSORB scaffold were still taking aspirin and clopidogrel at 3 years, which may have resulted in a low rate of scaffold thrombosis.

There was a sharp increase in the Kaplan-Meier curves for TLF, PoCE, MACE, and ID-TLR at 12 months postprocedure. One explanation is that most patients with ischemic symptoms were angiographically examined and revascularized at the 12-month follow-up according to the protocol, although symptoms of angina were routinely recorded. We closely monitored these patients during the study. They were admitted to hospitals if severe chest pain or MI occurred. These curves remained steady from year 1 through year 3 in SES-treated patients, suggesting no added adverse events occurred. However, there was a clear but mild increase in the curves of clinical events beyond one year in the XINSORB-treated population, although the rates of TLF, PoCE, MACE, and ID-TLR is still lower than those in the SES arm. It was believed there was a so-called "late catch-up phenomenon" after first-generation drugeluting stent (DES) implantation (22). The incidence of very late stent thrombosis and TLR was significantly higher after DES implantation than after bare-metal stent (BMS) implantation on long-term follow-up (23). Compared with patients not showing the late catch-up phenomenon, those exhibiting the late catch-up phenomenon showed slow but significant additive LLL occurring from the early to longterm follow-ups $(22,24)$. Also, neoatherosclerosis with a different morphological appearance was observed by optical coherence tomography (OCT) in the restenotic area $(25,26)$. We cannot conclude a "late catch-up phenomenon" in this study. The 5-year clinical results from the XINSORB firstin-human study showed relatively low rates of TLF and scaffold thrombosis (9). However, when we look at the long-term follow-up results of the serial ABSORB studies, we should know the potential risks of the very late failure of the XINSORB scaffold.

There were some limitations to this study. First, both simple and moderately complex lesions were treated in this study. The treatment of complex lesions with a BRS may be related to higher rates of scaffold thrombosis and target vessel MI (15). The XINSORB randomized clinical trial met the premarket approval requirements of the State Administration for Market Regulation in China. Patients and lesions with higher risks were excluded. Second, both the predilation of target lesions and the post-dilation of implanted scaffolds were mandatory. However, not all operators stuck to the protocol. Post dilation was performed significantly less for the metallic stent than the XINSORB scaffold, which may influence the interpretation of the study results. Third, a longer observation period must be included to verify the long-term efficacy and safety of the XINSORB scaffold. We previously reported the five-year intravascular imaging outcomes of the XINSORB scaffold in a first-in-man study (27). Although the lumen remained patent, the OCT findings showed that some struts were not fully absorbed. We must wait and see what will happen and how the vessels will heal after the struts are absorbed, and we need to confirm whether a "late catch-up phenomenon" will occur after XINSORB scaffold implantation. Finally, the sample size of this study was modest. More extensive studies with long-term follow-up data must estimate the incidence of late and very late events after XINSORB scaffold implantation. The impact of optimal implantation techniques should also be evaluated.

\section{Conclusions}

In this multicenter randomized clinical trial, the XINSORB SES showed similar efficacy and safety outcomes compared 
with the SES up to the 3-year follow-up. The rates of TLF and device thrombosis were low and comparable between the two arms. More extensive studies with more extended observation periods must evaluate the incidence of very late events after treatment with the XINSORB scaffold.

\section{Acknowledgments}

Funding: Grants funded this work from the National Natural Science Foundation of China (Nos. 81521001 and 81670319) and the National Key Research and Development Program (Nos. 2016YFC1102300 and 2016YFC1102303).

\section{Footnote}

Reporting Checklist: The authors have completed the CONSORT reporting checklist. Available at http://dx.doi. org/10.21037/atm-20-6739

Data Sharing Statement: Available at http://dx.doi. org/10.21037/atm-20-6739

Conflicts of Interest: All authors have completed the ICMJE uniform disclosure form (available at http://dx.doi. org/10.21037/atm-20-6739). The authors have no conflicts of interest to declare.

Ethical Statement: The authors are accountable for all aspects of the work in ensuring that questions related to the accuracy or integrity of any part of the work are appropriately investigated and resolved. All procedures performed in this study involving human participants were in accordance with the Declaration of Helsinki (as revised in 2013). The study was approved by Zhongshan Hospital (No. 2014-44). Written informed consent was obtained before patient enrollment.

Open Access Statement: This is an Open Access article distributed in accordance with the Creative Commons Attribution-NonCommercial-NoDerivs 4.0 International License (CC BY-NC-ND 4.0), which permits the noncommercial replication and distribution of the article with the strict proviso that no changes or edits are made and the original work is properly cited (including links to both the formal publication through the relevant DOI and the license). See: https://creativecommons.org/licenses/by-nc$\mathrm{nd} / 4.0 /$.

\section{References}

1. Stone GW, Gao R, Kimura T, et al. 1-year outcomes with the Absorb bioresorbable scaffold in patients with coronary artery disease: a patient-level, pooled meta-analysis. Lancet 2016;387:1277-89.

2. Geng X, Liu B, Liu J, et al. Interfacial tissue engineering of heart regenerative medicine based on soft cell-porous scaffolds. J Thorac Dis 2018;10:S2333-45.

3. Ali ZA, Gao R, Kimura T, et al. Three-Year Outcomes With the Absorb Bioresorbable Scaffold: IndividualPatient-Data Meta-Analysis From the ABSORB Randomized Trials. Circulation 2018;137:464-79.

4. Modolo R, Collet C, Onuma Y, et al. SYNTAX II and SYNTAX III trials: what is the take home message for surgeons? Ann Cardiothorac Surg 2018;7:470-82.

5. Gao R, Yang Y, Han Y, et al. Bioresorbable Vascular Scaffolds Versus Metallic Stents in Patients With Coronary Artery Disease: ABSORB China Trial. J Am Coll Cardiol 2015;66:2298-309.

6. Xu B, Yang Y, Han Y, et al. Comparison of everolimuseluting bioresorbable vascular scaffolds and metallic stents: three-year clinical outcomes from the ABSORB China randomised trial. EuroIntervention 2018;14:e554-61.

7. Wu Y, Shen L, Ge L, et al. Six-month outcomes of the XINSORB bioresorbable sirolimus-eluting scaffold in treating single de novo lesions in human coronary artery. Catheter Cardiovasc Interv 2016;87 Suppl 1:630-7.

8. Wu Y, Shen L, Wang Q, et al. Comparison of Acute Recoil Between Bioabsorbable Poly-L-lactic Acid XINSORB Scaffold and Metallic Stent in Porcine Model. J Biomed Biotechnol 2012;2012:413956.

9. Wu Y, Yin J, Chen J, et al. Final report of the 5-year clinical outcomes of the XINSORB bioresorbable sirolimus-eluting scaffold in the treatment of single de novo coronary lesions in a first-in-human study. Ann Transl Med 2020;8:1162.

10. Wu Y, Shen L, Yin J, et al. XINSORB randomized clinical trial. Twelve-month angiographic and clinical outcomes of the XINSORB bioresorbable sirolimus-eluting scaffold and a metallic stent in patients with coronary artery disease. Int J Cardiol 2019;293:61-6.

11. Han $Y, X u$ B, Jing Q, et al. A randomized comparison of novel biodegradable polymer- and durable polymercoated cobalt-chromium sirolimus-eluting stents. JACC Cardiovasc Interv 2014;7:1352-60.

12. Song L, Li J, Guan C, et al. I-LOVE-IT 2 Investigators. Randomized comparison of novel biodegradable polymer 
and durable polymer-coated cobalt-chromium sirolimuseluting stents: Three-Year Outcomes of the I-LOVE-IT 2 Trial. Catheter Cardiovasc Interv 2018;91:608-16.

13. Cutlip DE, Windecker S, Mehran R, et al. Academic Research Consortium. Clinical end points in coronary stent trials: a case for standardized definitions. Circulation 2007;115:2344-51.

14. Serruys PW, Chevalier B, Sotomi Y, et al. Comparison of an everolimus-eluting bioresorbable scaffold with an everolimus-eluting metallic stent for the treatment of coronary artery stenosis (ABSORB II): a 3 year, randomised, controlled, single-blind, multicentre clinical trial. Lancet 2016;388:2479-91.

15. Kereiakes DJ, Ellis SG, Metzger C, et al. ABSORB III Investigators. 3-Year Clinical Outcomes With EverolimusEluting Bioresorbable Coronary Scaffolds: The ABSORB III Trial. J Am Coll Cardiol 2017;70:2852-62.

16. Tijssen RYG, Kraak RP, Hofma SH, et al. Complete two-year follow-up with formal non-inferiority testing on primary outcomes of the AIDA trial comparing the Absorb bioresorbable scaffold with the XIENCE drugeluting metallic stent in routine PCI. EuroIntervention 2018;14:e426-33.

17. Stone GW, Ellis SG, Gori T, et al. Blinded outcomes and angina assessment of coronary bioresorbable scaffolds: 30 day and 1-year results from the ABSORB IV randomised trial. Lancet 2018;392:1530-40.

18. Gilard M, Barragan P, Noryani AAL, et al. 6- versus 24-month dual antiplatelet therapy after implantation of drug-eluting stents in patients nonresistant to aspirin: the randomized, multicenter ITALIC trial. J Am Coll Cardiol 2015;65:777-86.

19. Mauri L, Kereiakes DJ, Yeh RW, et al. Twelve or 30 months of dual antiplatelet therapy after drug-eluting stents. N Engl J Med 2014;371:2155-66.

20. Ge J. Bioresorbable vascular scaffold for the treatment of

Cite this article as: Wu Y, Yao Z, Yin J, Chen J, Qian J, Shen L, Ge L, Ge J; on behalf of the XINSORB randomized clinical trial. Three-year clinical outcomes of a sirolimus-eluting bioresorbable scaffold (XINSORB) and a metallic stent to treat coronary artery stenosis. Ann Transl Med 2020;8(22):1489. doi: 10.21037/atm-20-6739 coronary in-stent restenosis: New dawn or frost on snow? Catheter Cardiovasc Interv 2018;92:678-9.

21. Chevalier B, Cequier A, Dudek D, et al. Four-year followup of the randomised comparison between an everolimuseluting bioresorbable scaffold and an everolimus-eluting metallic stent for the treatment of coronary artery stenosis (ABSORB II Trial). EuroIntervention 2018;13:1561-4.

22. Yang TH, Kim DI, Jin HY, et al. "Angiographic late catch-up" phenomenon after sirolimus-eluting stent implantation. Int J Cardiol 2012;160:48-52.

23. Natsuaki M, Morimoto T, Furukawa Y, et al. CREDOKyoto PCI/CABG registry cohort-2 investigators. Late adverse events after implantation of sirolimus-eluting stent and bare-metal stent: long-term (5-7 years) follow-up of the Coronary Revascularization Demonstrating Outcome study-Kyoto registry Cohort-2. Circ Cardiovasc Interv 2014;7:168-79.

24. Iijima R, Araki T, Nagashima Y, et al. Incidence and predictors of the late catch-up phenomenon after drugeluting stent implantation. Int J Cardiol 2013;168:2588-92.

25. Yamaguchi H, Arikawa R, Takaoka J, et al. Association of morphologic characteristics on optical coherence tomography and angiographic progression patterns of late restenosis after drug-eluting stent implantation. Cardiovasc Revasc Med 2015;16:32-5.

26. Naganuma T, Fujino Y, Tahara S, et al. Extremely Late Catch-Up Phenomenon After First-Generation SirolimusEluting Stent in the Left Main Stem: Insights From Optical Coherence Tomography. JACC Cardiovasc Interv 2015;8:e237-9.

27. Wu Y, Shen L, Yin J, et al. 5 Years of Serial Intravascular Imaging Outcomes of XINSORB Sirolimus-Eluting Bioresorbable Vascular Scaffold. JACC Cardiovasc Interv 2019;12:602-3.

(English Language Editor: J. Chapnick) 